\title{
A Patient's Guide to Nuclear Medicine Procedures: English-Spanish
}

J.C. Mas

Reston, VA: SNM, 2008, 88 pages, $\$ 99$

A Patient's Guide to Nuclear Medicine Procedures: English-Spanish, by Juan Mas, is a flip-chart book containing over 35 common nuclear medicine procedures explained in layperson's terms. As the title indicates, the descriptions are in both English and Spanish.

I found the book to give an appropriately worded general but complete description of each procedure. I particularly liked the descriptions of myocardial perfusion imaging and lung scanning. Even with all the variability in these studies, the author was able to broach these topics and give complete but generic descriptions. Mr. Mas has also conveniently left blanks throughout the descriptions to allow the nuclear medicine department to specify and individualize certain nuclear medicine procedures, such as return times for bone scans.

The flip-chart format is made of durable material with convenient tabs that separate the different procedures. The type size is large enough to allow for easy reading. The book can be placed on a table in such a way that the patient can read the description in Spanish while the technologist sees the English description for the same procedure.

Besides the flip-chart model, a pocket-sized model that fits into a lab-coat pocket is also available. The pocketsized version is perfect for discussions with inpatients.

A Patient's Guide to Nuclear Medicine Procedures: English-Spanish by Juan Mas alleviates the need to have numerous brochures. This book gathers descriptions of most nuclear medicine procedures into one convenient location. The text is accurately translated and is easy to read and understand. I would recommend this book to all nuclear medicine departments. It is an absolute must-have for any nuclear medicine department with Spanish-speaking patients.

Sara Garcia Johnson, MBA, CNMT, NCT VA Hospital San Diego San Diego, California 